\title{
Combination of combretastatin A4 phosphate and doxorubicin-containing liposomes affects growth of B16-F10 tumors
}

\author{
Iwona Mitrus $\bowtie$, Aleksander Sochanik, Tomasz Cichoń and Stanisław Szala \\ Maria Skłodowska-Curie Memorial Cancer Center and Institute of Oncology, Gliwice, Poland
}

Received: 17 December, 2008; revised: 06 February, 2009; accepted: 11 March 2009

available on-line: 14 March, 2009

\begin{abstract}
The study aimed to check the effectiveness of anticancer therapy combining a vascular-disruptive drug (combretastatin phosphate, CA4P) and a liposomal formulation of a chemotherapeutic (doxorubicin). CA4P was synthesized in our laboratory according to a previously described procedure. The antivascular drug and long-circulating doxorubicin-loaded liposomes were used to treat B16-F10 murine melanoma experimental tumors. Seventy-four hours after drug administration, a decrease in the number of tumor blood vessels was apparent and necrotic areas within tumors were visible. Combination therapy consisting of alternate administrations of CA4P and liposomal doxorubicin yielded greater inhibition of tumor growth than monotherapies alone. The best therapeutic results were obtained with the antivascular drug administered intratumorally every second day at $50 \mathrm{mg} / \mathrm{kg}$ body mass. In the case of combined therapy, the best results were obtained when the vascular-disruptive agent (CA4P) and the antineoplastic agent (liposomal doxorubicin) were administered in alternation.
\end{abstract}

Keywords: CA4P, liposomes, doxorubicin, combined therapy

\section{INTRODUCTION}

A major problem facing cancer therapy stems from limited drug access to the inside of the tumor (Minchinton \& Tannock, 2006). As opposed to the tumor periphery which has adequate blood supply, the tumor core is poorly vascularized (Sivridis et al., 2003; Ribatti et al., 2007). Blood vessels inside tumors are malformed; they are leaky, tortuous, have numerous strictures, dead-ends, etc. This results in inadequate supply of oxygen and nutrients to the tumor inside and also in poor penetrability of drugs. Vascular-disruptive agents selectively destroy tumor vasculature and cut off oxygen and nutrient supplies to the tumor (Thorpe, 2004; Tozer et al., 2005). Cancer cells then undergo necrotic death. However, cells forming external tumor layer are likely to survive vascular-disruptive therapies. In time this leads to tumor regression. But, from the therapeutic perspective, the fact that the outer layer of tumor cells is supported by a quasi-adequate blood supply re- sults in chemotherapeutic substances also having easier access to such cells. More refined antitumor therapies therefore combine two different approaches based on: vascular-disruptive agents and chemotherapeutics to enhance eradication of neoplastic cells surviving at the tumor edge. Combining antivascular therapy with chemotherapy is expected to result in improved therapeutic outcome compared to either approach separately (Shi et al., 2006).

Combretastatin is a low-molecular-weight drug exerting antivascular action (Tozer et al., 2002; West \& Price, 2004; Kanthou \& Tozer, 2007). It inhibits polymerization of tubulin and precludes termination of cell division. Since one of the distinguishing features of tumor endothelium is its intense proliferation, combretastatin acts mainly upon tumor blood vessels. Usually, combretastatin is used as a water soluble prodrug (combretastatin phosphate, CA4P).

In order to destroy the outlying cancer cells refractory to antivascular therapy we used doxorubicin, an anthracycline-type drug, which acts by

${ }^{\square}$ Corresponding author: Iwona Mitrus, Maria Skłodowska-Curie Memorial Cancer Center and Institute of Oncology, Wybrzeże Armii Krajowej 15, 44-101 Gliwice, Poland; tel: (48) 32278 9698; fax: (48) 32278 9846; e-mail: mitrus@io.gliwice.pl Abbreviations: B16-F10, murine melanoma cells; CA4P, combretastatin A4 phosphate; FBS, fetal bovine serum; EPR, enhanced permeability and retention; H\&E, hematoxylin and eosin staining; PBS, phosphate-buffered saline. 
intercalating into the DNA helix, inhibiting topoisomerase II and arresting the cell cycle (Soloman \& Gabizon, 2008). The drug was transferred into experimentally-induced murine tumors with the help of liposomes (Gabizon et al., 2006; Soloman \& Gabizon, 2008). Owing to extended liposome half-life in circulation, doxorubicin bioavailability is increased and its toxicity diminished. Suitable size of extruded liposomes (about $100 \mathrm{~nm}$ ) allows their selective localization within the tumor. This so-called EPR (enhanced permeability and retention) effect is possible because of leaky tumor capillaries and their tortuous course and results in mechanical retention of extravasated liposomes within the tumor core (Gabizon et al., 2006).

\section{MATERIALS AND METHODS}

Synthesis of combretastatin CA4 phosphate. Synthesis of combretastatin CA4 was carried out according to Gaukroger and coworkers (2001). The phosphate group was introduced into combretastatin using our own protocol. Briefly, phosphoryl oxychloride was reacted ( $1 \mathrm{~h}$ at room temperature) with combretastatin $\left(290 \mu \mathrm{l} \mathrm{POCl}_{3}\right.$ per $100 \mathrm{mg}$ CA4$)$ in anhydrous benzene $(5 \mathrm{ml})$ in the presence of $30 \mu \mathrm{l}$ pyridine. The precipitated pyridine hydrochloride was filtered off and the remaining solution concentrated and chromatographed using a silica gel column and ethyl acetate/ethanol $(40: 1, \mathrm{v} / \mathrm{v})$ as eluant. In order to carry out hydrolysis the obtained product was dissolved in water and placed in a rotary evaporator (to eliminate $\mathrm{HCl}$ ). After $1 \mathrm{~h}$ the resulting suspension was frozen and lyophilized. In order to obtain a sodium salt, to the weighed out residue an aliquot of $\mathrm{NaOH}$ solution was added ( 2 moles $\mathrm{NaOH}$ per 1 mol combretastatin).

The correct structure of the synthesized compound was confirmed using ${ }^{1} \mathrm{H}$ NMR and mass spectrometry (ESI).

Liposome preparation. Liposomes were made of distearoylphosphatidylcholine (DSPC), cholesterol and distearoylphosphatidylethanolamine conjugated with $\mathrm{N}$-carbamoylmetoxy-polyethyleneglycol /nontargeted, liposomes, 9:5:1 ( $\mathrm{mol} / \mathrm{mol})$. Liposomes were extruded (100 nm-pore polycarbonate membrane) and dialyzed into 10\% sucrose. Doxorubicin was internalized (in excess of 95\%) using transmembrane $\mathrm{pH}$ gradient, as described by Zhou and coworkers (2002), with minor modifications. Phospholipid concentration was assessed by measuring total phosphorus (Bartlett, 1958). Size and size distribution of liposome dispersions was measured by photon correlation spectroscopy (PCS) which indicated a discrete mono-modal, log-normal size distribution in the range between 20 to over $200 \mathrm{~nm}$ in diameter with the majority of liposomes not exceeding $130 \mathrm{~nm}$ in size.

Animal studies. Mice C57Bl/6 6-8 week old were inoculated with B16-F10 cells $\left(2 \times 10^{6}\right.$ per $100 \mu \mathrm{l}$ $\mathrm{PBS}^{-} /$animal). The therapy was started on the $6^{\text {th }}$ day following inoculation, when tumors reached $4-5 \mathrm{~mm}$ in diameter. The size of the tumors was measured with calipers and volume was calculated from the following formula: $\mathrm{V}=(\text { width })^{2} \times$ length $\times 0.52$.

CA4P administration. Varying doses of CA4P were administered intratumorally (in $100 \mu \mathrm{l} \quad 0.9 \%$ $\mathrm{NaCl}$ ), intraperitoneally or intravenously (in $200 \mu \mathrm{l}$ of $0.9 \% \mathrm{NaCl}$ ).

Liposome administration. Liposomes were administered intravenously (200 $\mu \mathrm{l}$ of liposome emulsion in $10 \%$ sucrose) into the tail vein of $\mathrm{C} 57 \mathrm{Bl} / 6$ tumor-bearing mice.

Vessel count. The number of vessels present in tumors was determined according to the protocol described by Siemann and coworkers (2002). A single dose of CA4P $(30 \mathrm{mg} / \mathrm{kg}$ body mass) was administered once to animals bearing about $6-7 \mathrm{~mm}$ tumors. After $0,24,48$ and $72 \mathrm{~h}$ the mice were administered Sigma's Hoechst $33342(40 \mathrm{mg} / \mathrm{kg}$ body mass) in $100 \mu \mathrm{l}$ of $0.9 \% \mathrm{NaCl}$ and were sacrificed. The tumors were frozen in liquid nitrogen and cryostat sections prepared. Tumor sections were then photographed under the microscope in such a way that they would fill out the whole viewing area and the number of vessels per unit section area was determined.

Hematoxylin and eosin staining (H\&E). CA4P (50 mg/kg body mass, in $100 \mu \mathrm{l} \mathrm{NaCl}$ ) was injected intratumorally every $48 \mathrm{~h}$. Animals were sacrificed and tumor material collected $24 \mathrm{~h}$ after one, two or three injections of the drug. Tumor tissue was paraffin-embedded and H\&E staining of tumor sections performed.

\section{RESULTS AND DISCUSSION}

In the initial stage of the study we optimized combretastatin dose and mode of administration. The experiments were initiated when murine melanoma tumors reached approx. 4-5 mm. CA4P was administered either intravenously or intratumorally (50 mg/kg body mass), or intraperitoneally (50 or $200 \mathrm{mg} / \mathrm{kg}$ body mass). The therapeutic effect was assessed based on tumor growth inhibition in the treated groups as compared to control (Fig. 1). The strongest inhibition was observed in the case of direct intratumoral injection.

During the course of the investigation changes in the appearance of tumor blood vessels were observed following combretastatin administration which attested to their progressive destruction (Fig. 2A and 


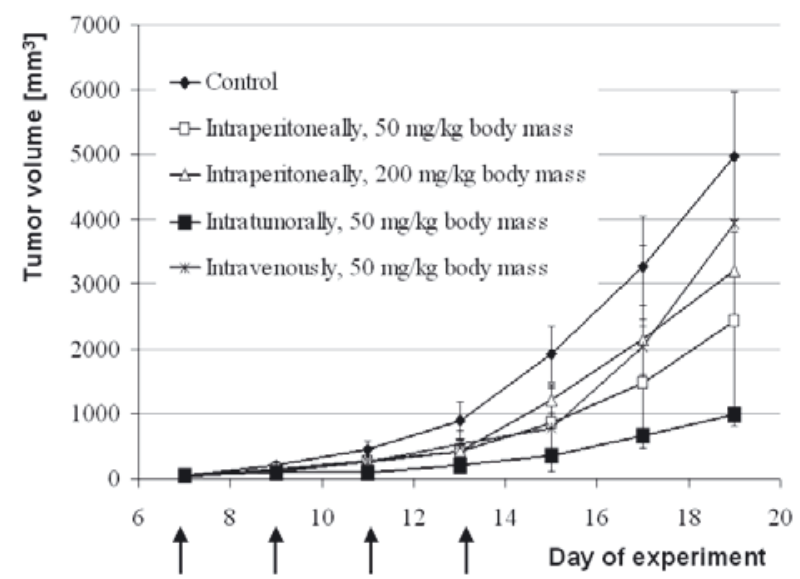

Figure 1. Optimization of CA4P administration.

Various ways of administering CA4P (arrows) to mice with formed experimental tumors were examined. In case of intraperitoneal administration two different drug doses were used; otherwise $50 \mathrm{mg} / \mathrm{kg}$ body mass was used (see inset). The drug was dissolved in $100 \mu \mathrm{l} 0.9 \% \mathrm{NaCl}$. Each experimental group consisted of 5 animals.
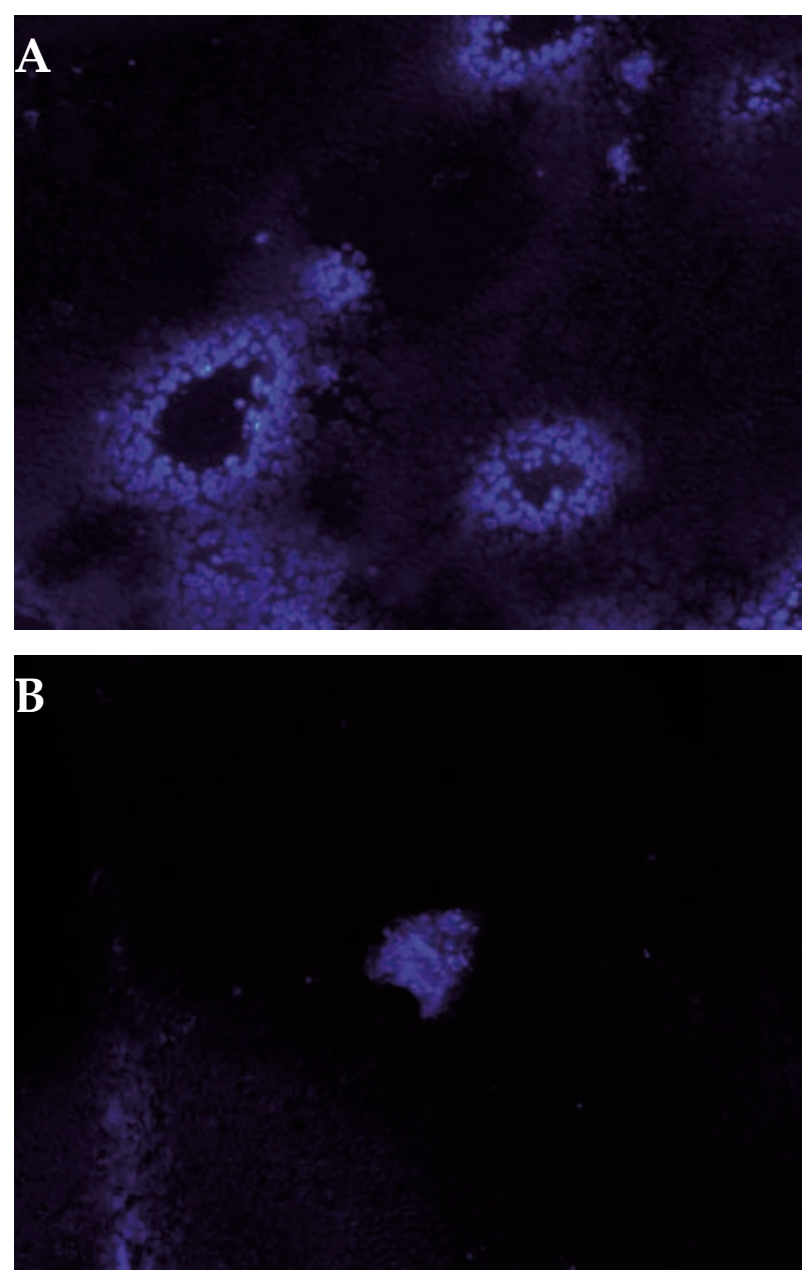

Figure 2. Tumor blood vessels (visualized with Hoechst reagent) $48 \mathrm{~h}$ post administration of $0.9 \% \mathrm{NaCl}$ (A) and CA4P (B).

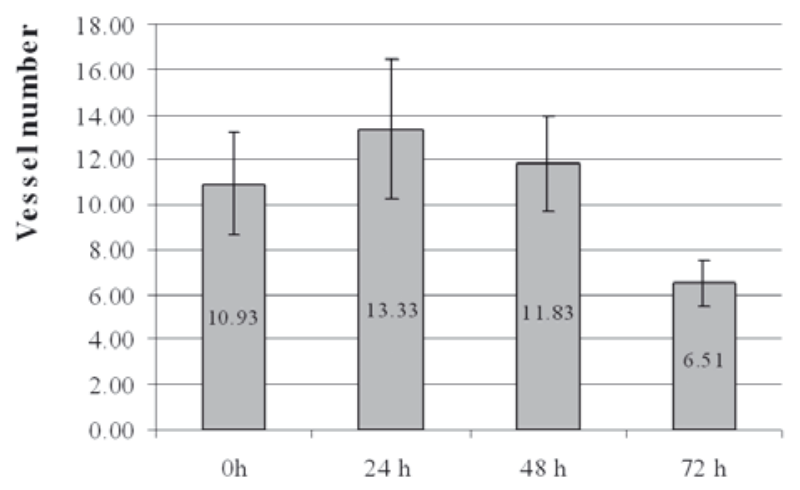

Figure 3. Count of the number of vessels in B16-F10 tumor sections following administration of a single CA4P dose.

Mice harboring about 6-7 $\mathrm{mm}$ tumors were administered (once) CA4P at $50 \mathrm{mg} / \mathrm{kg}$ body mass. At a predetermined time ( $\mathrm{X}$ axis) after inoculation the mice were given Hoechst reagent and were sacrificed. Tumor material was collected and sections prepared. The number of vessels was counted using a microscope. For each time point shown the average was calculated from 4 tumors (at least 8 sections from different tumor layers).

2B). Use of Hoechst reagent has allowed to determine changes in vessel number in combretastatin-exposed tumors. The vessels were scored at $0,24,48$ and 72 $\mathrm{h}$ post single drug dose administration. A two-fold drop in the number of tumor blood vessels was observed only $72 \mathrm{~h}$ after drug delivery (Fig. 3), although already at $48 \mathrm{~h}$ changes in the structure of vascular network were apparent. This is a significantly longer time period than that reported by Siemann and coworkers (2002; 2007). In their study, a similar effect was observed already a few hours post drug administration. It is, perhaps, an effect specific for murine melanoma tumors.

We also investigated (by H\&E staining of tumor sections) the effect of combretastatin upon the morphology of the tumors. Large necrotic areas were found together with destroyed blood vessels (Fig. 4A and 4B). The accompanying drug, doxorubicin, was administered to tumor-bearing animals using intravenously-injected long-lived liposomes (Pastorino et al., 2003).

Since, according to reference data, the maximum tolerated dose should not exceed $400 \mu \mathrm{g} /$ mouse per week, we checked that and also lower doses (Fig. 5). We found that the therapeutic effect is similar for all doses tested, but related toxicity (assessed as body weight loss) is directly dose-dependent. Accordingly, in further studies we used aliquots of liposomes equivalent to $50 \mu \mathrm{g}$ of doxorubicin/animal per dose. We did not use free doxorubicin in these further experiments, as preliminary toxicity trials clearly demonstrated liposomal doxorubicin to be superior to free drug (not shown). 

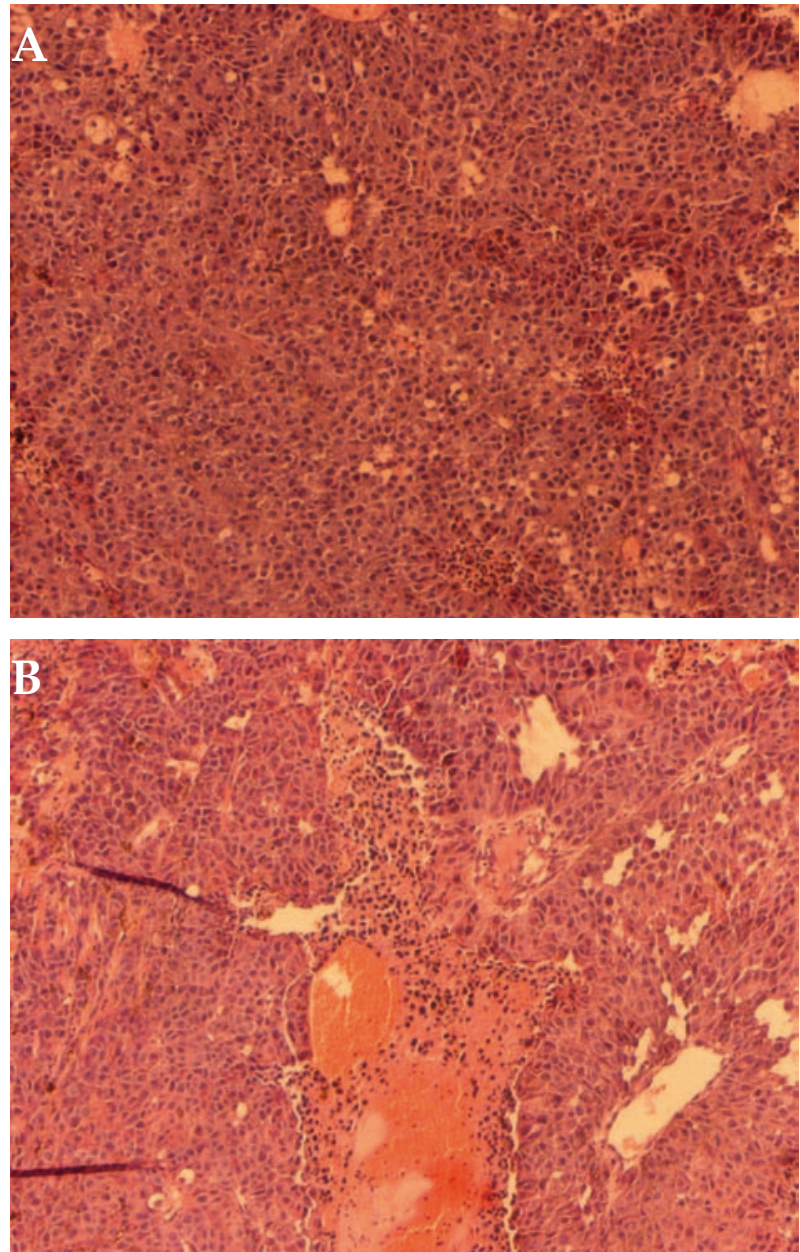

Figure 4. Tumor section, $24 \mathrm{~h}$ after second administration of $0.9 \% \mathrm{NaCl}$ (A) and CA4P (B).

In trials involving combined therapy we decided to reduce both doxorubicin dose (down to $40 \mu \mathrm{g} /$ mouse per injection) as well as that of combretastatin (down to $30 \mathrm{mg} / \mathrm{kg}$ body mass) because of enhanced drug toxicities (inferred from body weight loss) observed in such trials.

In our study of combination therapy with vascular-disruptive agent and liposomal doxorubicin we also checked various sequences of administering both drugs (Horsman \& Siemann, 2006). According to the most often practiced approach to combination therapy involving antivascular and chemotherapeutic agents the vascular compartment is targeted first (Fig. 6). Only then the chemotherapeutic is introduced to destroy viable cells remaining at the tumor rim. In our case this approach was not effective as we did not notice differences between groups receiving combined therapy and those receiving liposomal doxorubicin only (not shown).

Since we assumed that with the use of liposomes the EPR effect is sizeable, we decided to investigate whether there would be a benefit from administering liposomes alternatingly with vascular-disrup-

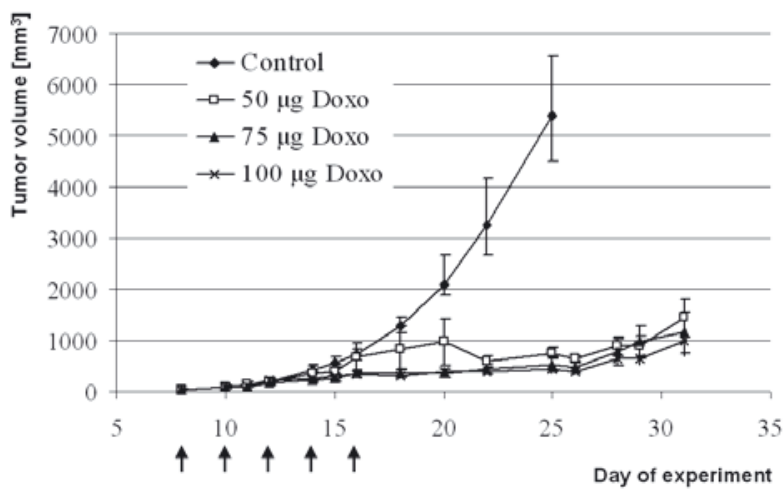

Figure 5. Optimization of doxorubicin administration.

Mice with preformed experimental tumors were tail-injected with liposome formulations containing doxorubicin. In each group 5 administrations (every second day) were performed, each consisting of $100 \mu \mathrm{l}$ of liposomal emulsion carrying the indicated amounts of the drug (inset). Each experimental group consisted of 5 animals.

tive therapy. This might help entrapping the chemotherapeutic within the tumor core and prevent its escape from the intended site of action. As mentioned before, a decrease in the number of tumor blood vessels became apparent only after about $72 \mathrm{~h}$ post combretastatin administration which means that during this period liposomes have access to the tumor core. Subsequent destruction of tumor vasculature is likely to enhance the EPR effect. Such an approach indeed improved the therapeutic outcome.

To conclude, the "standard" approach to combination anticancer therapy involving chemotherapeutics and vascular-disruptive agents might not always be an optimal solution. Intricacies of a particular tumor model play an important role in a therapeutic outcome.

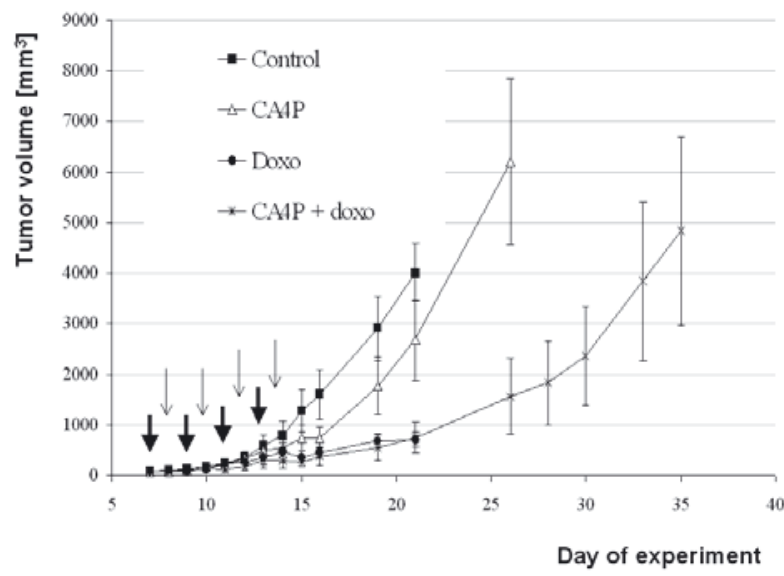

Figure 6. Inhibition of tumor growth following administration of CA4P and doxorubicin-containing liposomes. CA4P was administered intratumorally $(30 \mathrm{mg} / \mathrm{kg}$ body mass) in $100 \mu \mathrm{l} 0.9 \% \mathrm{NaCl}$ (small arrows). Liposomes with doxorubicin were administered intravenously (40 $\mathrm{mg}$ of drug/kg body mass) in $200 \mu \mathrm{l} 10 \%$ sucrose (large arrows). Each experimental group consisted of 6 animals. 


\section{Acknowledgements}

The study was supported by a Commissioned Grant (No. PBZ-KBN-091/P05/2003) and a Grant (No. 2P05A 07428), both from the Polish Ministry of Science and Higher Education.

\section{REFERENCES}

Bartlett GR (1958) Phosphorus assay in column chromatography. J Biol Chem 234: 466-468.

Gabizon AA, Shmeeda H, Zalipsky S (2006) Pros and cons of the liposome platform in cancer drug targeting. J $\mathrm{Li}$ posome Res 16: 175-183.

Gaukroger K, Hadfield JA, Hepworth LA, Lawrence NJ, McGown AT (2001) Novel syntheses of cis and trans isomers of combretastatin A-4. J Org Chem 66: 81358138.

Horsman MR, Siemann DW (2006) Pathophysiologic effects of vascular-targeting agents and the implications for combination with conventional therapies. Cancer Res 66: 11520-11539.

Kanthou C, Tozer GM (2007) Tumour targeting by microtubule-depolymerizing vascular disrupting agents. Expert Opin Ther Targets 11: 1443-1457.

Minchinton AI, Tannock IF (2006) Drug penetration in solid tumours. Nat Rev Cancer 6: 583-592.

Pastorino F, Brignole C, Marimpietri D, Cilli M, Gambini C, Ribatti D, Longhi R, Allen TM, Corti A, Ponzoni M (2003) Vascular damage and anti-angiogenic effects of tumor vessel-targeted liposomal chemotherapy. Cancer Res 63: 7400-7409.
Ribatti D, Nico B, Crivellato E, Vacca A (2007) The structure of the vascular network of tumors. Cancer Lett 248: 18-23.

Salmon BA, Siemann DW (2007) Characterizing the tumor response to treatment with combretastatin A4 phosphate. Int J Radiat Oncol Biol Phys 68: 211-217.

Shi W, Horsman MR, Siemann DW (2006) Combined modality approaches using vasculature-disrupting agents. In Vascular-targeted Therapies in Oncology, Siemann DW, ed, pp 123-136. Chichester. John Wiley \& Sons Ltd.

Siemann DW, Mercer E, Lepler S, Rojiani AM (2002) Vascular targeting agents enhance chemotherapeutic agent activities in solid tumor therapy. Int J Cancer 99: 1-6.

Sivridis E, Giatromanolaki A, Koukourakis MI (2003) The vascular network of tumours - what is it not for? J Pathol 201: 173-180.

Soloman R, Gabizon AA (2008) Clinical pharmacology of liposomal anthracyclines: focus on pegylated liposomal doxorubicin. Clin Lymphoma Myeloma 8: 21-32.

Thorpe PE (2004) Vascular targeting agents as cancer therapeutics. Clin Cancer Res 10: 415-427.

Tozer GM, Kanthou C, Baguley BC (2005) Disrupting tumour blood vessels. Nat Rev Cancer 5: 423-435.

Tozer GM, Kanthou C, Parkins CS, Hill SA (2002) The biology of the combretastatins as tumour vascular targeting agents. Int J Exp Pathol 83: 21-38.

West CM, Price P (2004) Combretastatin A4 phosphate. Anticancer Drugs 215: 179-187.

Zhou R, Mazurchuk R, Straubinger RM (2002) Antivasculature effects of doxorubicin-containing liposomes in an intracranial rat brain tumor model. Cancer Res 62: 2561-2566. 